\title{
III-V Plasmonic Solar Cells: Targeting Absorption Enhancements Close to the GaAs Band Edge
}

\author{
N. P. Hylton ${ }^{1}$, V. Giannini ${ }^{1}$, D. Vercruysse ${ }^{2}$, P. Van Dorpe ${ }^{2}$, K.-H. Lee ${ }^{1}$, X. Li ${ }^{1}$, N. J. Ekins-Daukes ${ }^{1}$ and S. \\ A. Maier ${ }^{1}$ \\ 1. Experimental Solid State Group, Physics Department, Imperial College London, London SW7 $2 A Z$, UK \\ 2. imec vzw, Kapeldreef 75, B-3001 Leuven, Belgium
}

Research into photovoltaics has attracted a great deal of attention in recent years since it represents a realistic solution to the so-called 'energy crisis'. To date however, solar cells have suffered from relatively high cost efficiency $(\$ / W p)[1,2]$, limiting their commercial uptake. Recent research has therefore focused on the need to improve solar cell efficiencies to make photovoltaics a viable alternative to fossil fuels. One area of investigation is in the application of plasmonic nanoparticles in order to enhance the absorption of light in solar cells [1-3]. Such an enhancement of the photoabsorption may allow the fabrication of structures in which the optical absorption depth is significantly larger than the thickness of the absorbing material, reducing the amount of material required and hence the cost of production [1,3].

In particular we aim to build upon the success of high efficiency GaAs and c-Si based photovoltaic cells by targeting the spectral region close to the band edge, where the absorption strength in conventional GaAs solar cells is poor. Quantum well (QW) solar cells have been employed in an attempt to extend the spectral absorption in this regime; however they require many QWs grown in a stack, leading to long growth times and an increased use of semiconductor material. Employing plasmonic nanoparticles to enhance the absorption in this spectral region may relax this requirement, permitting the use of fewer QWs while achieving the same quantum efficiency.
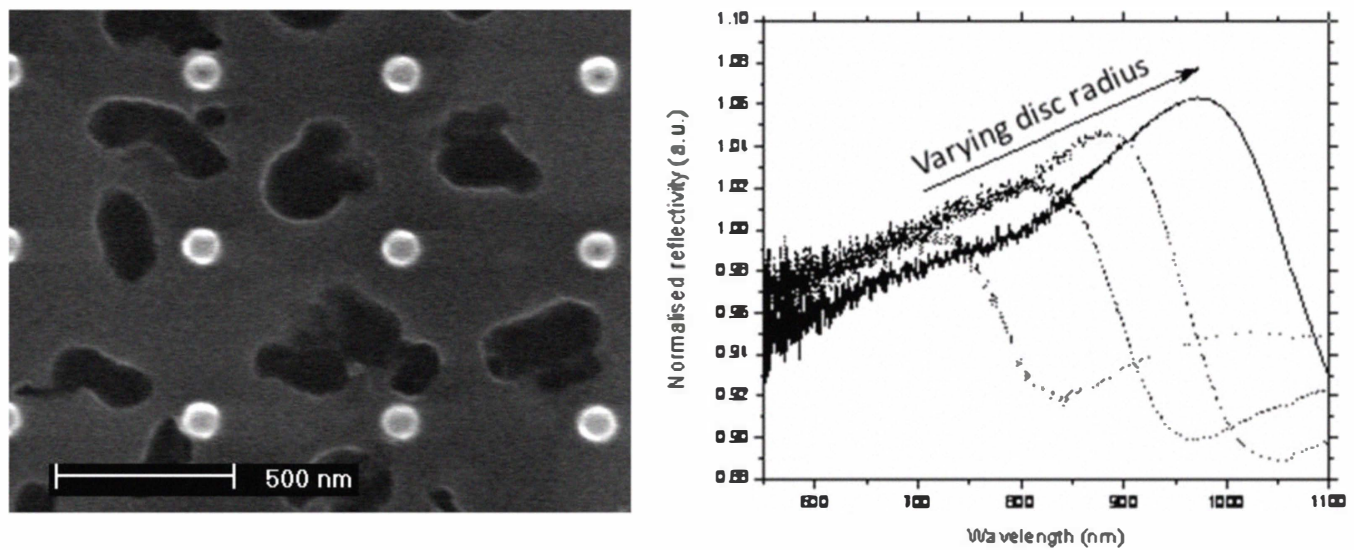

Fig. 1 (a) SEM image showing a periodic array of Au nanoparticles on GaAs and (b) FTIR spectra of a series of Au disc arrays on GaAs, clearly showing localised surface Plasmon resonances whose peak wavelength is a function of the disc dimensions.

Au nanoparticles were fabricated on the surface of absorbing GaAs layers using electron beam lithography (see Fig. 1 (a)). We will present the results of dark-field and Fourier-transform infra-red (FTIR) spectroscopy as a function of the nanoparticle size and shape. The results are supported by theoretical simulations and demonstrate that by careful adjustment of the nanoparticle structure and dimensions we can tune (see Fig. 1 (b)) the localised surface Plasmon resonance to the desired wavelength. Furthermore, we show evidence of grazing diffracted orders associated with the periodic nature of the arrays, which can also be tuned by controlling the spacing between nanoparticles. Such periodic nanoparticle arrays therefore give rise to two methods of absorption enhancement that can be employed in the same structure. Finally, we will extend the study to include nanoparticle arrays on full solar cell structures.

\section{References}

[1] Harry A Atwater and Albert Polman, "Plasmonics for improved photovoltaic devices" Nature Mat. 9, 205-13 (2010).

[2] Katsuaki Tanabe, "A Review of Ultrahigh Efficiency III-V Semiconductor Compound Solar Cells: Multijunction Tandem, Lower Dimensional, Photonic Up/Down Conversion and Plasmonic Nanometallic Structures" Energies 2, 504-530 (2009).

[3] F. J. Beck, A. Polman, and K. R. Catchpole, "Tunable light trapping for solar cells using localized surface plasmons" Journal Of Applied Physics 105, 114310 (2009). 